\title{
Existence results for multi-term fractional differential inclusions
}

\author{
Sotiris K Ntouyas ${ }^{1,2}$, Sina Etemad ${ }^{3}$ and Jessada Tariboon ${ }^{4 *}$
}

"Correspondence:

jessadat@kmutnb.ac.th

${ }^{4}$ Nonlinear Dynamic Analysis Research Center, Department of Mathematics, Faculty of Applied Science, King Mongkut's University of Technology North Bangkok, Bangkok, 10800, Thailand Full list of author information is available at the end of the article

\begin{abstract}
In this paper, we study the existence of solutions for a new class of boundary value problems for nonlinear multi-term fractional differential inclusions. Our main result relies on the multi-valued form of Krasnoselskii's fixed point theorem. An illustrative example is also presented.
\end{abstract}

MSC: 34A08; 34A60; 34B10; 34B15

Keywords: fractional differential inclusion; boundary value problem; existence; fixed point theorem

\section{Introduction and preliminaries}

In this paper we study the existence of solutions for the following multi-term fractional differential inclusions:

$$
\begin{aligned}
{ }^{c} D^{\alpha} u(t) \in & F\left(t, u(t), u^{\prime}(t), u^{\prime \prime}(t),{ }^{c} D^{q_{1}} u(t), \ldots,{ }^{c} D^{q_{k}} u(t)\right) \\
& +G\left(t, u(t), u^{\prime}(t), u^{\prime \prime}(t),{ }^{c} D^{q_{1}} u(t), \ldots,{ }^{c} D^{q_{k}} u(t)\right)
\end{aligned}
$$

supplemented with boundary conditions

$$
u(0)=0, \quad u^{\prime}(0)=-u(1)-u^{\prime}(1), \quad u^{\prime \prime}(0)=-u^{\prime \prime}(1)-{ }^{c} D^{p} u(1)
$$

where ${ }^{c} D^{\alpha},{ }^{c} D^{q_{i}}$ denote the Caputo fractional derivatives, $2<\alpha \leq 3,1<q_{i} \leq 2, i=$ $1,2, \ldots, k, t \in J:=[0,1], 1<p \leq 2, k \geq 1$, and $F, G: J \times \mathbb{R}^{k+3} \rightarrow \mathcal{P}(\mathbb{R})$ are multifunctions.

Many of published papers about fractional differential equations and inclusions apply the fixed point theory for proving the existence results. For instance, one can find a lot of papers in this field (see [1-25] and the references therein).

Let $\alpha>0, n-1<\alpha<n, n=[\alpha]+1$, and $u \in C([a, b], \mathbb{R})$. The Caputo derivative of fractional of order $\alpha$ for the function $u$ is defined by ${ }^{c} D^{\alpha} u(t)=\frac{1}{\Gamma(n-\alpha)} \int_{0}^{t}(t-\tau)^{n-\alpha-1} u^{(n)}(\tau) d \tau$ (see for more details [11, 23, 25-27]). Also, the Riemann-Liouville fractional order integral of the function $u$ is defined by $I^{\alpha} u(t)=\frac{1}{\Gamma(\alpha)} \int_{0}^{t} \frac{u(\tau)}{(t-\tau)^{1-\alpha}} d \tau(t>0)$ whenever the integral exists [11, 23, 25-27]. In [28], it has been proved that the general solution of the fractional differential equation ${ }^{c} D^{\alpha} u(t)=0$ is given by $u(t)=c_{0}+c_{1} t+c_{2} t^{2}+\cdots+c_{n-1} t^{n-1}$, where $c_{0}, \ldots, c_{n-1}$ are real constants and $n=[\alpha]+1$. Also, for each $T>0$ and $u \in C([0, T])$ we

(c) 2015 Ntouyas et al.; licensee Springer. This article is distributed under the terms of the Creative Commons Attribution 4.0 International License (http://creativecommons.org/licenses/by/4.0/), which permits unrestricted use, distribution, and reproduction in any medium, provided you give appropriate credit to the original author(s) and the source, provide a link to the Creative Commons license, and indicate if changes were made. 
have

$$
I^{\alpha c} D^{\alpha} u(t)=u(t)+c_{0}+c_{1} t+c_{2} t^{2}+\cdots+c_{n-1} t^{n-1},
$$

where $c_{0}, \ldots, c_{n-1}$ are real constants and $n=[\alpha]+1[28]$.

Now, we review some definitions and notations as regards multifunctions $[29,30]$.

For a normed space $(X,\|\cdot\|)$, let $\mathcal{P}_{\mathrm{cl}}(X)=\{Y \in \mathcal{P}(X): Y$ is closed $\}, \mathcal{P}_{\mathrm{b}}(X)=\{Y \in$ $\mathcal{P}(X): Y$ is bounded $\}, \mathcal{P}_{\text {cp }}(X)=\{Y \in \mathcal{P}(X): Y$ is compact $\}$, and $\mathcal{P}_{\text {cp,cv }}(X)=\{Y \in \mathcal{P}(X)$ : $Y$ is compact and convex $\}, \mathcal{P}_{\mathrm{b}, \mathrm{cl}, \mathrm{cv}}(X)=\{Y \in \mathcal{P}(X): Y$ is bounded, closed, and convex $\}$. A multi-valued map $G: X \rightarrow \mathcal{P}(X)$ is convex (closed) valued if $G(x)$ is convex (closed) for all $x \in X$. The map $G$ is bounded on bounded sets if $G(\mathbb{B})=\bigcup_{x \in \mathbb{B}} G(x)$ is bounded in $X$ for all $\mathbb{B} \in \mathcal{P}_{\mathrm{b}}(X)$ (i.e., $\sup _{x \in \mathbb{B}}\{\sup \{|y|: y \in G(x)\}\}<\infty$ ). $G$ is called upper semicontinuous (u.s.c.) on $X$ if for each $x_{0} \in X$, the set $G\left(x_{0}\right)$ is a nonempty closed subset of $X$, and if for each open set $N$ of $X$ containing $G\left(x_{0}\right)$, there exists an open neighborhood $\mathcal{N}_{0}$ of $x_{0}$ such that $G\left(\mathcal{N}_{0}\right) \subseteq N$. $G$ is said to be completely continuous if $G(\mathbb{B})$ is relatively compact for every $\mathbb{B} \in \mathcal{P}_{\mathrm{b}}(X)$. If the multi-valued map $G$ is completely continuous with nonempty compact values, then $G$ is u.s.c. if and only if $G$ has a closed graph, i.e., $u_{n} \rightarrow u_{*}, y_{n} \rightarrow y_{*}, y_{n} \in G\left(u_{n}\right)$ imply $y_{*} \in G\left(u_{*}\right)$. $G$ has a fixed point if there is $x \in X$ such that $x \in G(x)$. The fixed point set of the multi-valued operator $G$ will be denoted by Fix $G$. A multi-valued map $G: J \rightarrow \mathcal{P}_{\mathrm{cl}}(\mathbb{R})$ is said to be measurable if for every $y \in \mathbb{R}$, the function $t \mapsto d(y, G(t))=\inf \{|y-z|: z \in G(t)\}$ is measurable.

Consider the Pompeiu-Hausdorff metric $H_{d}: \mathcal{P}(X) \times \mathcal{P}(X) \rightarrow \mathbb{R} \cup\{\infty\}$ given by

$$
H_{d}(A, B)=\max \left\{\sup _{a \in A} d(a, B), \sup _{b \in B} d(A, b)\right\},
$$

where $d(A, b)=\inf _{a \in A} d(a ; b)$ and $d(a, B)=\inf _{b \in B} d(a ; b)$. A multi-valued operator $N: X \rightarrow$ $\mathcal{P}_{\mathrm{cl}}(X)$ is called contraction if there exists $\gamma \in(0,1)$ such that $H_{d}(N(x), N(y)) \leq \gamma d(x, y)$ for each $x, y \in X$.

We say that $F: J \times \mathbb{R}^{k+3} \rightarrow \mathcal{P}(\mathbb{R})$ is a Carathéodory multifunction if $t \mapsto F\left(t, u_{1}, \ldots, u_{k+3}\right)$ is measurable for all $u_{i} \in \mathbb{R}$ and $\left(u_{1}, \ldots, u_{k+3}\right) \mapsto F\left(t, u_{1}, \ldots, u_{k+3}\right)$ is upper semi-continuous for almost all $t \in J[29,31]$. Also, a Carathéodory multifunction $F: J \times \mathbb{R}^{k+3} \rightarrow \mathcal{P}(\mathbb{R})$ is called $L^{1}$-Carathéodory if for each $\rho>0$ there exists $\phi_{\rho} \in L^{1}\left(J, \mathbb{R}^{+}\right)$such that

$$
\left\|F\left(t, u_{1}, \ldots, u_{k+3}\right)\right\|=\sup _{t \in J}\left\{|s|: s \in F\left(t, u_{1}, \ldots, u_{k+3}\right)\right\} \leq \phi_{\rho}(t)
$$

for all $\left|u_{1}\right|, \ldots,\left|u_{k+3}\right| \leq \rho$ and for almost all $t \in J[29,31]$.

Define the set of selections of $F$ and $G$ at $u \in C(J, \mathbb{R})$ by

$$
S_{F, u}:=\left\{v \in L^{1}(J, \mathbb{R}): v(t) \in F\left(t, u(t), u^{\prime}(t), u^{\prime \prime}(t),{ }^{c} D^{q_{1}} u(t), \ldots,{ }^{c} D^{q_{k}} u(t)\right)\right\}
$$

and

$$
S_{G, u}:=\left\{v_{1} \in L^{1}(J, \mathbb{R}): v_{1}(t) \in G\left(t, u(t), u^{\prime}(t), u^{\prime \prime}(t),{ }^{c} D^{q_{1}} u(t), \ldots,{ }^{c} D^{q_{k}} u(t)\right)\right\}
$$

for almost all $t \in J$. If $F$ is an arbitrary multifunction, then it has been proved that $S_{F}(u) \neq \emptyset$ for all $u \in C(J, X)$ if $\operatorname{dim} X<\infty$ [32]. 
The graph of a function $F$ is the set $\operatorname{Gr}(F)=\{(x, y) \in X \times Y: y \in F(x)\}$ [29]. The graph $\operatorname{Gr}(F)$ of $F: X \rightarrow \mathcal{P}_{\mathrm{cl}}(Y)$ is said to be a closed subset of $X \times Y$, if for every sequence $\left\{u_{n}\right\}_{n \in \mathbb{N}} \subset X$ and $\left\{y_{n}\right\}_{n \in \mathbb{N}} \subset Y$, when $n \rightarrow \infty, u_{n} \rightarrow u_{0}, y_{n} \rightarrow y_{0}$, and $y_{n} \in F\left(u_{n}\right)$, then $y_{0} \in F\left(u_{0}\right)[29]$.

We will use the following lemmas and theorem in our main result.

Lemma 1.1 ([29], Proposition 1.2) If $F: X \rightarrow \mathcal{P}_{\mathrm{cl}}(Y)$ is u.s.c., then $\operatorname{Gr}(F)$ is a closed subset of $X \times Y$. Conversely, if $F$ is completely continuous and has a closed graph, then it is upper semi-continuous.

Lemma 1.2 ([32]) Let $X$ be a separable Banach space. Let $F:[0,1] \times X^{k+3} \rightarrow \mathcal{P}_{\mathrm{cp}, \mathrm{cv}}(X)$ be an $L^{1}$-Carathéodory function. Then the operator

$$
\Theta \circ S_{F}: C(J, X) \rightarrow \mathcal{P}_{\mathrm{cp}, \mathrm{cv}}(C(J, X)), \quad x \mapsto\left(\Theta \circ S_{F}\right)(x)=\Theta\left(S_{F, x}\right)
$$

is a closed graph operator.

Theorem 1.3 ([33], Krasnoselskii's fixed point theorem) Let $X$ be a Banach space, $Y \in$ $\mathcal{P}_{\mathrm{b}, \mathrm{cl}, \mathrm{cv}}(X)$ and $A, B: Y \rightarrow \mathcal{P}_{\mathrm{cp}, \mathrm{cv}}(X)$ two multi-valued operators. If the following conditions are satisfied:

(i) $A y+B y \subset Y$ for all $y \in Y$;

(ii) $A$ is a contraction;

(iii) $B$ is u.s.c. and compact,

then there exists $y \in Y$ such that $y \in A y+B y$.

\section{Main results}

Now, we are ready to prove our main result. Let $X=\left\{u: u, u^{\prime}, u^{\prime \prime},{ }^{c} D^{q_{i}} u \in C(J, \mathbb{R}), i=\right.$ $1,2, \ldots, k\}$. Then $(X,\|\cdot\|)$ endowed with the norm

$$
\|u\|=\sup _{t \in J}|u(t)|+\sup _{t \in J}\left|u^{\prime}(t)\right|+\sup _{t \in J}\left|u^{\prime \prime}(t)\right|+\sup _{t \in J}\left|{ }^{c} D^{q_{i}} u(t)\right| \quad(i=1, \ldots, k)
$$

is a Banach space [34].

We need the following auxiliary lemma. See also $[35,36]$.

Lemma 2.1 Let $y \in C(J, \mathbb{R})$ and $u \in C^{2}([0,1], \mathbb{R})$ is a solution to the fractional boundary value problem

$$
\left\{\begin{array}{l}
{ }^{c} D^{\alpha} u(t)=y(t), \\
u(0)=0, \quad u^{\prime}(0)=-u(1)-u^{\prime}(1), \quad u^{\prime \prime}(0)=-u^{\prime \prime}(1)-{ }^{c} D^{p} u(1),
\end{array}\right.
$$

then

$$
\begin{aligned}
u(t)= & \int_{0}^{t} \frac{(t-s)^{\alpha-1}}{\Gamma(\alpha)} y(s) d s-\frac{t}{3} \int_{0}^{1} \frac{(1-s)^{\alpha-1}}{\Gamma(\alpha)} y(s) d s-\frac{t}{3} \int_{0}^{1} \frac{(1-s)^{\alpha-2}}{\Gamma(\alpha-1)} y(s) d s \\
& +\left(t-t^{2}\right) \Delta \int_{0}^{1} \frac{(1-s)^{\alpha-3}}{\Gamma(\alpha-2)} y(s) d s+\left(t-t^{2}\right) \Delta \int_{0}^{1} \frac{(1-s)^{\alpha-p-1}}{\Gamma(\alpha-p)} y(s) d s,
\end{aligned}
$$

and vice versa, where $\Delta=\frac{\Gamma(3-p)}{4 \Gamma(3-p)+2} \neq 0$. 
Proof It is well known that the solution of equation ${ }^{c} D^{\alpha} u(t)=y(t)$ can be written as

$$
u(t)=\int_{0}^{t} \frac{(t-s)^{\alpha-1}}{\Gamma(\alpha)} y(s) d s+c_{0}+c_{1} t+c_{2} t^{2},
$$

where $c_{0}, c_{1}, c_{2} \in \mathbb{R}$. Then we get

$$
\begin{aligned}
& u^{\prime}(t)=\int_{0}^{t} \frac{(t-s)^{\alpha-2}}{\Gamma(\alpha-1)} y(s) d s+c_{1}+2 c_{2} t, \\
& u^{\prime \prime}(t)=\int_{0}^{t} \frac{(t-s)^{\alpha-3}}{\Gamma(\alpha-2)} y(s) d s+2 c_{2}
\end{aligned}
$$

and

$$
{ }^{c} D^{p} u(t)=\int_{0}^{t} \frac{(t-s)^{\alpha-p-1}}{\Gamma(\alpha-p)} y(s) d s+c_{2} \frac{2 t^{2-p}}{\Gamma(3-p)} \quad(1<p \leq 2) .
$$

By using the boundary value conditions, we obtain $c_{0}=0$ and

$$
\begin{aligned}
c_{1}= & -\frac{1}{3} \int_{0}^{1} \frac{(1-s)^{\alpha-1}}{\Gamma(\alpha)} y(s) d s-\frac{1}{3} \int_{0}^{1} \frac{(1-s)^{\alpha-2}}{\Gamma(\alpha-1)} y(s) d s \\
& +\Delta \int_{0}^{1} \frac{(1-s)^{\alpha-3}}{\Gamma(\alpha-2)} y(s) d s+\Delta \int_{0}^{1} \frac{(1-s)^{\alpha-p-1}}{\Gamma(\alpha-p)} y(s) d s
\end{aligned}
$$

and

$$
c_{2}=-\Delta \int_{0}^{1} \frac{(1-s)^{\alpha-3}}{\Gamma(\alpha-2)} y(s) d s-\Delta \int_{0}^{1} \frac{(1-s)^{\alpha-p-1}}{\Gamma(\alpha-p)} y(s) d s .
$$

Substituting the values of $c_{0}, c_{1}$, and $c_{2}$ in (2.3) we get (2.2).

Conversely, applying the operator ${ }^{c} D^{\alpha}$ on (2.2) and taking into account (2.1), it follows that ${ }^{c} D^{\alpha} u(t)=y(t)$. From (2.2) it is easily to verify that the boundary conditions $u(0)=0$, $u^{\prime}(0)=-u(1)-u^{\prime}(1), u^{\prime \prime}(0)=-u^{\prime \prime}(1)-{ }^{c} D^{p} u(1)$ are satisfied. This establishes the equivalence between (2.1) and (2.2). The proof is completed.

Definition 2.2 A function $u \in C^{2}([0,1], \mathbb{R})$ is called a solution for the problem (1.1)-(1.2) if it satisfies the boundary value conditions $u(0)=0, u^{\prime}(0)=-u(1)-u^{\prime}(1)$, and $u^{\prime \prime}(0)=$ $-u^{\prime \prime}(1)-{ }^{c} D^{p} u(1)$, there exist functions $v, v_{1} \in L^{1}(J, \mathbb{R})$ such that $v(t) \in F\left(t, u(t), u^{\prime}(t), u^{\prime \prime}(t)\right.$, $\left.{ }^{c} D^{q_{1}} u(t), \ldots,{ }^{c} D^{q_{k}} u(t)\right), v_{1}(t) \in G\left(t, u(t), u^{\prime}(t), u^{\prime \prime}(t),{ }^{c} D^{q_{1}} u(t), \ldots,{ }^{c} D^{q_{k}} u(t)\right)$ for almost all $t \in J$ and

$$
\begin{aligned}
u(t)= & \int_{0}^{t} \frac{(t-s)^{\alpha-1}}{\Gamma(\alpha)} v(s) d s-\frac{t}{3} \int_{0}^{1} \frac{(1-s)^{\alpha-1}}{\Gamma(\alpha)} v(s) d s \\
& -\frac{t}{3} \int_{0}^{1} \frac{(1-s)^{\alpha-2}}{\Gamma(\alpha-1)} v(s) d s+\left(t-t^{2}\right) \Delta \int_{0}^{1} \frac{(1-s)^{\alpha-3}}{\Gamma(\alpha-2)} v(s) d s \\
& +\left(t-t^{2}\right) \Delta \int_{0}^{1} \frac{(1-s)^{\alpha-p-1}}{\Gamma(\alpha-p)} v(s) d s \\
& +\int_{0}^{t} \frac{(t-s)^{\alpha-1}}{\Gamma(\alpha)} v_{1}(s) d s-\frac{t}{3} \int_{0}^{1} \frac{(1-s)^{\alpha-1}}{\Gamma(\alpha)} v_{1}(s) d s
\end{aligned}
$$




$$
\begin{aligned}
& -\frac{t}{3} \int_{0}^{1} \frac{(1-s)^{\alpha-2}}{\Gamma(\alpha-1)} v_{1}(s) d s+\left(t-t^{2}\right) \Delta \int_{0}^{1} \frac{(1-s)^{\alpha-3}}{\Gamma(\alpha-2)} v_{1}(s) d s \\
& +\left(t-t^{2}\right) \Delta \int_{0}^{1} \frac{(1-s)^{\alpha-p-1}}{\Gamma(\alpha-p)} v_{1}(s) d s .
\end{aligned}
$$

Remark 2.3 For the sake of brevity, we set

$$
\begin{aligned}
& \Lambda_{1}=\frac{4}{3 \Gamma(\alpha+1)}+\frac{1}{3 \Gamma(\alpha)}+\frac{\Delta}{4 \Gamma(\alpha-1)}+\frac{\Delta}{4 \Gamma(\alpha-p+1)}, \\
& \Lambda_{2}=\frac{4}{3 \Gamma(\alpha)}+\frac{1}{3 \Gamma(\alpha+1)}+\frac{\Delta}{\Gamma(\alpha-1)}+\frac{\Delta}{\Gamma(\alpha-p+1)}, \\
& \Lambda_{3}=\frac{1+2 \Delta}{\Gamma(\alpha-1)}+\frac{2 \Delta}{\Gamma(\alpha-p+1)},
\end{aligned}
$$

and, for each $i=1, \ldots, k$,

$$
\Lambda_{4}^{i}=\frac{1}{\Gamma\left(\alpha-q_{i}+1\right)}+\frac{2 \Delta}{\Gamma\left(3-q_{i}\right) \Gamma(\alpha-1)}+\frac{2 \Delta}{\Gamma\left(3-q_{i}\right) \Gamma(\alpha-p+1)} .
$$

Also in the following we use the notation $\|x\|_{\infty}=\sup \{|x(t)|: t \in J\}$.

\section{Theorem 2.4 Suppose that:}

$\left(\mathrm{H}_{1}\right) F: J \times \mathbb{R}^{k+3} \rightarrow \mathcal{P}_{\mathrm{cp}, c}(\mathbb{R})$ is a multifunction and $G: J \times \mathbb{R}^{k+3} \rightarrow \mathcal{P}_{\mathrm{cp}, c}(\mathbb{R})$ is a Carathéodory multifunction;

$\left(\mathrm{H}_{2}\right)$ there exist continuous functions $p, m: J \rightarrow(0, \infty)$ such that $t \mapsto F\left(t, w_{1}, w_{2}, w_{3}, z_{1}\right.$, $\left.\ldots, z_{k}\right)$ is measurable and

$$
\left\|F\left(t, w_{1}, w_{2}, w_{3}, z_{1}, \ldots, z_{k}\right)\right\| \leq m(t), \quad\left\|G\left(t, w_{1}, w_{2}, w_{3}, z_{1}, \ldots, z_{k}\right)\right\| \leq p(t) ;
$$

$\left(\mathrm{H}_{3}\right)$ there exists a continuous function $h: J \rightarrow(0, \infty)$ such that

$$
\begin{aligned}
& H_{d}\left(F\left(t, w_{1}, w_{2}, w_{3}, z_{1}, \ldots, z_{k}\right), F\left(t, w_{1}^{\prime}, w_{2}^{\prime}, w_{3}^{\prime}, z_{1}^{\prime}, \ldots, z_{k}^{\prime}\right)\right) \\
& \quad \leq h(t)\left[\sum_{i=1}^{3}\left|w_{i}-w_{i}^{\prime}\right|+\sum_{i=1}^{k}\left|z_{i}-z_{i}^{\prime}\right|\right]
\end{aligned}
$$

for all $t \in J$ and for each $w_{1}, w_{2}, w_{3}, z_{1}, \ldots, z_{k}, w_{1}^{\prime}, w_{2}^{\prime}, w_{3}^{\prime}, z_{1}^{\prime}, \ldots, z_{k}^{\prime} \in \mathbb{R}$.

If

$$
L:=\|h\|_{\infty}\left(\Lambda_{1}+\Lambda_{2}+\Lambda_{3}+\Lambda_{4}^{i}\right)<1
$$

for $i=1,2, \ldots, k$, where the $\Lambda_{j}(j=1, \ldots, 4)$ are defined in (2.5)-(2.8), then the inclusion problem (1.1)-(1.2) has at least one solution.

Proof We define the subset $Y$ of $X$ by $Y=\{u \in X:\|u\| \leq M\}$, where

$$
M=\left(\|p\|_{\infty}+\|m\|_{\infty}\right)\left(\Lambda_{1}+\Lambda_{2}+\Lambda_{3}+\Lambda_{4}^{i}\right) \quad(i=1, \ldots, k)
$$


It is clear that $Y$ is closed, bounded, and convex subset of Banach space $X$. We define the multi-valued operators $A, B: Y \rightarrow \mathcal{P}(X)$ such that for some $v \in S_{F, u}$,

$$
\begin{aligned}
A(u)= & \left\{u \in X: u(t)=\int_{0}^{t} \frac{(t-s)^{\alpha-1}}{\Gamma(\alpha)} v(s) d s-\frac{t}{3} \int_{0}^{1} \frac{(1-s)^{\alpha-1}}{\Gamma(\alpha)} v(s) d s\right. \\
& -\frac{t}{3} \int_{0}^{1} \frac{(1-s)^{\alpha-2}}{\Gamma(\alpha-1)} v(s) d s+\left(t-t^{2}\right) \Delta \int_{0}^{1} \frac{(1-s)^{\alpha-3}}{\Gamma(\alpha-2)} v(s) d s \\
& \left.+\left(t-t^{2}\right) \Delta \int_{0}^{1} \frac{(1-s)^{\alpha-p-1}}{\Gamma(\alpha-p)} v(s) d s\right\},
\end{aligned}
$$

and for some $v_{1} \in S_{G, u}$,

$$
\begin{aligned}
B(u)= & \left\{u \in X: u(t)=\int_{0}^{t} \frac{(t-s)^{\alpha-1}}{\Gamma(\alpha)} v_{1}(s) d s-\frac{t}{3} \int_{0}^{1} \frac{(1-s)^{\alpha-1}}{\Gamma(\alpha)} v_{1}(s) d s\right. \\
& -\frac{t}{3} \int_{0}^{1} \frac{(1-s)^{\alpha-2}}{\Gamma(\alpha-1)} v_{1}(s) d s+\left(t-t^{2}\right) \Delta \int_{0}^{1} \frac{(1-s)^{\alpha-3}}{\Gamma(\alpha-2)} v_{1}(s) d s \\
& \left.+\left(t-t^{2}\right) \Delta \int_{0}^{1} \frac{(1-s)^{\alpha-p-1}}{\Gamma(\alpha-p)} v_{1}(s) d s\right\} .
\end{aligned}
$$

In this way, the fractional differential inclusion (1.1)-(1.2) is equivalent to the inclusion problem $u \in A u+B u$. We show that the multi-valued operators $A$ and $B$ satisfy the conditions of Theorem 1.3 on $Y$.

First, we show that the operators $A$ and $B$ define the multi-valued operators $A, B: Y \rightarrow$ $\mathcal{P}_{\text {cp,cv }}(X)$. First we prove that $A$ is compact-valued on $Y$. Note that the operator $A$ is equivalent to the composition $\mathcal{L} \circ S_{F}$, where $\mathcal{L}$ is the continuous linear operator on $L^{1}(J, \mathbb{R})$ into $X$, defined by

$$
\begin{aligned}
\mathcal{L}(v)(t)= & \int_{0}^{t} \frac{(t-s)^{\alpha-1}}{\Gamma(\alpha)} v(s) d s-\frac{t}{3} \int_{0}^{1} \frac{(1-s)^{\alpha-1}}{\Gamma(\alpha)} v(s) d s \\
& -\frac{t}{3} \int_{0}^{1} \frac{(1-s)^{\alpha-2}}{\Gamma(\alpha-1)} v(s) d s+\left(t-t^{2}\right) \Delta \int_{0}^{1} \frac{(1-s)^{\alpha-3}}{\Gamma(\alpha-2)} v(s) d s \\
& +\left(t-t^{2}\right) \Delta \int_{0}^{1} \frac{(1-s)^{\alpha-p-1}}{\Gamma(\alpha-p)} v(s) d s .
\end{aligned}
$$

Suppose that $u \in Y$ is arbitrary and let $\left\{v_{n}\right\}$ be a sequence in $S_{F, u}$. Then, by definition of $S_{F, u}$, we have $v_{n}(t) \in F\left(t, u(t), u^{\prime}(t), u^{\prime \prime}(t),{ }^{c} D^{q_{1}} u(t), \ldots,{ }^{c} D^{q_{k}} u(t)\right)$ for almost all $t \in J$. Since $F\left(t, u(t), u^{\prime}(t), u^{\prime \prime}(t),{ }^{c} D^{q_{1}} u(t), \ldots,{ }^{c} D^{q_{k}} u(t)\right)$ is compact for all $t \in J$, there is a convergent subsequence of $\left\{v_{n}(t)\right\}$ (we denote it by $\left\{v_{n}(t)\right\}$ again) that converges in measure to some $v(t) \in S_{F, u}$ for almost all $t \in J$. On the other hand, $\mathcal{L}$ is continuous, so $\mathcal{L}\left(v_{n}\right)(t) \rightarrow \mathcal{L}(v)(t)$ pointwise on $J$.

In order to show that the convergence is uniform, we have to show that $\left\{\mathcal{L}\left(v_{n}\right)\right\}$ is an equi-continuous sequence. Let $t_{1}, t_{2} \in J$ with $t_{1}<t_{2}$. Then we have

$$
\begin{aligned}
& \left|\mathcal{L}\left(v_{n}\right)\left(t_{2}\right)-\mathcal{L}\left(v_{n}\right)\left(t_{1}\right)\right| \\
& \quad \leq\left|\frac{1}{\Gamma(\alpha)} \int_{0}^{t_{1}}\left[\left(t_{2}-s\right)^{\alpha-1}-\left(t_{1}-s\right)^{\alpha-1}\right] v_{n}(s) d s+\frac{1}{\Gamma(\alpha)} \int_{t_{1}}^{t_{2}}\left(t_{2}-s\right)^{\alpha-1} v_{n}(s) d s\right|
\end{aligned}
$$




$$
\begin{aligned}
& +\frac{\left|t_{2}-t_{1}\right|}{3 \Gamma(\alpha)} \int_{0}^{1}(1-s)^{\alpha-1}\left|v_{n}(s)\right| d s+\frac{\left|t_{2}-t_{1}\right|}{3 \Gamma(\alpha-1)} \int_{0}^{1}(1-s)^{\alpha-2}\left|v_{n}(s)\right| d s \\
& +\frac{\Delta\left|\left[\left(t_{2}-t_{1}\right)-\left(t_{2}^{2}-t_{1}^{2}\right)\right]\right|}{\Gamma(\alpha-2)} \int_{0}^{1}(1-s)^{\alpha-3}\left|v_{n}(s)\right| d s \\
& +\frac{\Delta\left|\left[\left(t_{2}-t_{1}\right)-\left(t_{2}^{2}-t_{1}^{2}\right)\right]\right|}{\Gamma(\alpha-p)} \int_{0}^{1}(1-s)^{\alpha-p-1}\left|v_{n}(s)\right| d s \\
& \leq\|m\|_{\infty}\left\{\frac{\left|t_{2}^{\alpha}-t_{1}^{\alpha}\right|}{\Gamma(\alpha+1)}+\frac{\left|t_{2}-t_{1}\right|}{3 \Gamma(\alpha+1)}+\frac{\left|t_{2}-t_{1}\right|}{3 \Gamma(\alpha)}+\frac{\Delta\left|\left[\left(t_{2}-t_{1}\right)-\left(t_{2}^{2}-t_{1}^{2}\right)\right]\right|}{\Gamma(\alpha-1)}\right. \\
& \left.+\frac{\Delta\left|\left[\left(t_{2}-t_{1}\right)-\left(t_{2}^{2}-t_{1}^{2}\right)\right]\right|}{\Gamma(\alpha-p+1)}\right\} .
\end{aligned}
$$

Continuing this process, we have

$$
\left|\left(\mathcal{L}^{\prime}\left(v_{n}\right)\left(t_{2}\right)\right)-\left(\mathcal{L}^{\prime}\left(v_{n}\right)\left(t_{1}\right)\right)\right| \leq\|m\|_{\infty}\left\{\frac{\left|t_{2}^{\alpha-1}-t_{1}^{\alpha-1}\right|}{\Gamma(\alpha)}+\frac{\Delta\left|t_{2}-t_{1}\right|}{\Gamma(\alpha-1)}+\frac{\Delta\left|t_{2}-t_{1}\right|}{\Gamma(\alpha-p+1)}\right\}
$$

and

$$
\begin{aligned}
\left|\left(\mathcal{L}^{\prime \prime}\left(v_{n}\right)\left(t_{2}\right)\right)-\left(\mathcal{L}^{\prime \prime}\left(v_{n}\right)\left(t_{1}\right)\right)\right| \leq & \mid \frac{1}{\Gamma(\alpha-2)} \int_{0}^{t_{1}}\left[\left(t_{2}-s\right)^{\alpha-3}-\left(t_{1}-s\right)^{\alpha-3}\right] v_{n}(s) d s \\
& +\frac{1}{\Gamma(\alpha-2)} \int_{t_{1}}^{t_{2}}\left(t_{2}-s\right)^{\alpha-3} v_{n}(s) d s \mid \\
\leq & \|m\|_{\infty} \frac{\left|t_{2}^{\alpha-2}-t_{1}^{\alpha-2}\right|}{\Gamma(\alpha-1)},
\end{aligned}
$$

and, finally, for every $i=1, \ldots, k$,

$$
\begin{aligned}
& \left|\left({ }^{c} D^{q_{i}} \mathcal{L}\left(v_{n}\right)\left(t_{2}\right)\right)-\left({ }^{c} D^{q_{i}} \mathcal{L}\left(v_{n}\right)\left(t_{1}\right)\right)\right| \\
& \quad \leq\|m\|_{\infty}\left\{\frac{\left|t_{2}^{\alpha-q_{i}}-t_{1}^{\alpha-q_{i}}\right|}{\Gamma\left(\alpha-q_{i}+1\right)}+\frac{2 \Delta\left|t_{2}^{2-q_{i}}-t_{1}^{2-q_{i}}\right|}{\Gamma\left(3-q_{i}\right) \Gamma(\alpha-1)}+\frac{2 \Delta\left|t_{2}^{2-q_{i}}-t_{1}^{2-q_{i}}\right|}{\Gamma\left(3-q_{i}\right) \Gamma(\alpha-p+1)}\right\} .
\end{aligned}
$$

We see that the right-hand sides of the above inequalities tend to zero as $t_{2} \rightarrow t_{1}$. Thus, the sequence $\left\{\mathcal{L}\left(v_{n}\right)\right\}$ is equi-continuous and by using the Arzelá-Ascoli theorem, we see that there is a uniformly convergent subsequence. So, there is a subsequence of $\left\{v_{n}\right\}$ (we denote it again by $\left.\left\{v_{n}\right\}\right)$ such that $\mathcal{L}\left(v_{n}\right) \rightarrow \mathcal{L}(v)$. Note that $\mathcal{L}(v) \in \mathcal{L}\left(S_{F, u}\right)$. Hence, $A(u)=$ $\mathcal{L}\left(S_{F, u}\right)$ is compact for all $u \in Y$. So $A(u)$ is compact.

Now, we show that $A(u)$ is convex for all $u \in X$. Let $z_{1}, z_{2} \in A(u)$. We select $f_{1}, f_{2} \in S_{F, u}$ such that

$$
\begin{aligned}
z_{i}(t)= & \int_{0}^{t} \frac{(t-s)^{\alpha-1}}{\Gamma(\alpha)} f_{i}(s) d s-\frac{t}{3} \int_{0}^{1} \frac{(1-s)^{\alpha-1}}{\Gamma(\alpha)} f_{i}(s) d s \\
& -\frac{t}{3} \int_{0}^{1} \frac{(1-s)^{\alpha-2}}{\Gamma(\alpha-1)} f_{i}(s) d s+\left(t-t^{2}\right) \Delta \int_{0}^{1} \frac{(1-s)^{\alpha-3}}{\Gamma(\alpha-2)} f_{i}(s) d s \\
& +\left(t-t^{2}\right) \Delta \int_{0}^{1} \frac{(1-s)^{\alpha-p-1}}{\Gamma(\alpha-p)} f_{i}(s) d s, \quad i=1,2
\end{aligned}
$$


for almost all $t \in J$. Let $0 \leq \lambda \leq 1$. Then we have

$$
\begin{aligned}
{\left[\lambda z_{1}+(1-\lambda) z_{2}\right](t)=} & \int_{0}^{t} \frac{(t-s)^{\alpha-1}}{\Gamma(\alpha)}\left[\lambda f_{1}(s)+(1-\lambda) f_{2}(s)\right] d s \\
& -\frac{t}{3} \int_{0}^{1} \frac{(1-s)^{\alpha-1}}{\Gamma(\alpha)}\left[\lambda f_{1}(s)+(1-\lambda) f_{2}(s)\right] d s \\
& -\frac{t}{3} \int_{0}^{1} \frac{(1-s)^{\alpha-2}}{\Gamma(\alpha-1)}\left[\lambda f_{1}(s)+(1-\lambda) f_{2}(s)\right] d s \\
& +\left(t-t^{2}\right) \Delta \int_{0}^{1} \frac{(1-s)^{\alpha-3}}{\Gamma(\alpha-2)}\left[\lambda f_{1}(s)+(1-\lambda) f_{2}(s)\right] d s \\
& +\left(t-t^{2}\right) \Delta \int_{0}^{1} \frac{(1-s)^{\alpha-p-1}}{\Gamma(\alpha-p)}\left[\lambda f_{1}(s)+(1-\lambda) f_{2}(s)\right] d s .
\end{aligned}
$$

Since $F$ has convex values, $S_{F, u}$ is convex and $\lambda f_{1}(s)+(1-\lambda) f_{2}(s) \in S_{F, u}$. Thus

$$
\lambda z_{1}+(1-\lambda) z_{2} \in A(u)
$$

Consequently, $A$ is convex-valued. Similarly, $B$ is compact and convex-valued.

Here, we show that $A(u)+B(u) \subset Y$ for all $u \in Y$. Suppose that $u \in Y$ and $z_{1} \in A(u)$, $z_{2} \in B(u)$ are arbitrary elements. Choose $v_{1} \in S_{F, u}$ and $v_{2} \in S_{G, u}$ such that

$$
\begin{aligned}
z_{1}(t)= & \int_{0}^{t} \frac{(t-s)^{\alpha-1}}{\Gamma(\alpha)} v_{1}(s) d s-\frac{t}{3} \int_{0}^{1} \frac{(1-s)^{\alpha-1}}{\Gamma(\alpha)} v_{1}(s) d s \\
& -\frac{t}{3} \int_{0}^{1} \frac{(1-s)^{\alpha-2}}{\Gamma(\alpha-1)} v_{1}(s) d s+\left(t-t^{2}\right) \Delta \int_{0}^{1} \frac{(1-s)^{\alpha-3}}{\Gamma(\alpha-2)} v_{1}(s) d s \\
& +\left(t-t^{2}\right) \Delta \int_{0}^{1} \frac{(1-s)^{\alpha-p-1}}{\Gamma(\alpha-p)} v_{1}(s) d s
\end{aligned}
$$

and

$$
\begin{aligned}
z_{2}(t)= & \int_{0}^{t} \frac{(t-s)^{\alpha-1}}{\Gamma(\alpha)} v_{2}(s) d s-\frac{t}{3} \int_{0}^{1} \frac{(1-s)^{\alpha-1}}{\Gamma(\alpha)} v_{2}(s) d s \\
& -\frac{t}{3} \int_{0}^{1} \frac{(1-s)^{\alpha-2}}{\Gamma(\alpha-1)} v_{2}(s) d s+\left(t-t^{2}\right) \Delta \int_{0}^{1} \frac{(1-s)^{\alpha-3}}{\Gamma(\alpha-2)} v_{2}(s) d s \\
& +\left(t-t^{2}\right) \Delta \int_{0}^{1} \frac{(1-s)^{\alpha-p-1}}{\Gamma(\alpha-p)} v_{2}(s) d s
\end{aligned}
$$

for almost all $t \in J$. Hence, we get

$$
\begin{aligned}
\left|z_{1}(t)+z_{2}(t)\right| \leq & \frac{1}{\Gamma(\alpha)} \int_{0}^{t}(t-s)^{\alpha-1}\left(\left|v_{1}(s)\right|+\left|v_{2}(s)\right|\right) d s \\
& +\frac{|t|}{3 \Gamma(\alpha)} \int_{0}^{1}(1-s)^{\alpha-1}\left(\left|v_{1}(s)\right|+\left|v_{2}(s)\right|\right) d s \\
& +\frac{|t|}{3 \Gamma(\alpha-1)} \int_{0}^{1}(1-s)^{\alpha-2}\left(\left|v_{1}(s)\right|+\left|v_{2}(s)\right|\right) d s \\
& +\frac{\Delta\left|t-t^{2}\right|}{\Gamma(\alpha-2)} \int_{0}^{1}(1-s)^{\alpha-3}\left(\left|v_{1}(s)\right|+\left|v_{2}(s)\right|\right) d s
\end{aligned}
$$




$$
\begin{aligned}
& +\frac{\Delta\left|t-t^{2}\right|}{\Gamma(\alpha-p)} \int_{0}^{1}(1-s)^{\alpha-p-1}\left(\left|v_{1}(s)\right|+\left|v_{2}(s)\right|\right) d s \\
\leq & \left(\|p\|_{\infty}+\|m\|_{\infty}\right)\left\{\frac{4}{3 \Gamma(\alpha+1)}+\frac{1}{3 \Gamma(\alpha)}+\frac{\Delta}{4 \Gamma(\alpha-1)}+\frac{\Delta}{4 \Gamma(\alpha-p+1)}\right\} .
\end{aligned}
$$

Hence, $\sup _{t \in J}\left|z_{1}(t)+z_{2}(t)\right| \leq\left(\|p\|_{\infty}+\|m\|_{\infty}\right) \Lambda_{1}$. Also we have

$$
\left|z_{1}^{\prime}(t)+z_{2}^{\prime}(t)\right| \leq\left(\|p\|_{\infty}+\|m\|_{\infty}\right)\left\{\frac{4}{3 \Gamma(\alpha)}+\frac{1}{3 \Gamma(\alpha+1)}+\frac{\Delta}{\Gamma(\alpha-1)}+\frac{\Delta}{\Gamma(\alpha-p+1)}\right\},
$$

which implies that $\sup _{t \in J}\left|z_{1}^{\prime}(t)+z_{2}^{\prime}(t)\right| \leq\left(\|p\|_{\infty}+\|m\|_{\infty}\right) \Lambda_{2}$ and

$$
\left|z_{1}^{\prime \prime}(t)+z_{2}^{\prime \prime}(t)\right| \leq\left(\|p\|_{\infty}+\|m\|_{\infty}\right)\left\{\frac{1+2 \Delta}{\Gamma(\alpha-1)}+\frac{2 \Delta}{\Gamma(\alpha-p+1)}\right\}
$$

from which $\sup _{t \in J}\left|z_{1}^{\prime \prime}(t)+z_{2}^{\prime \prime}(t)\right| \leq\left(\|p\|_{\infty}+\|m\|_{\infty}\right) \Lambda_{3}$. Finally, for all $i=1, \ldots, k$, we have

$$
\begin{aligned}
& \left|{ }^{c} D^{q_{i}} z_{1}(t)+{ }^{c} D^{q_{i}} z_{2}(t)\right| \\
& \quad \leq\left(\|p\|_{\infty}+\|m\|_{\infty}\right)\left\{\frac{1}{\Gamma\left(\alpha-q_{i}+1\right)}+\frac{2 \Delta}{\Gamma\left(3-q_{i}\right) \Gamma(\alpha-1)}+\frac{2 \Delta}{\Gamma\left(3-q_{i}\right) \Gamma(\alpha-p+1)}\right\}
\end{aligned}
$$

and so $\sup _{t \in J}\left|{ }^{c} D^{q_{i}} z_{1}(t)+{ }^{c} D^{q_{i}} z_{2}(t)\right| \leq\left(\|p\|_{\infty}+\|m\|_{\infty}\right) \Lambda_{4}^{i}, i=1,2, \ldots, k$. Hence, it follows that

$$
\left\|z_{1}+z_{2}\right\| \leq\left(\|p\|_{\infty}+\|m\|_{\infty}\right)\left(\Lambda_{1}+\Lambda_{2}+\Lambda_{3}+\Lambda_{4}^{i}\right)=M, \quad i=1,2, \ldots, k
$$

Now, we show that the operator $B$ is compact on $Y$. To do this, it is enough to prove that $B(Y)$ is uniformly bounded and equi-continuous in $X$. Let $z \in B(Y)$ be arbitrary. For some $u \in Y$, choose $v_{1} \in S_{G, u}$ such that

$$
\begin{aligned}
z(t)= & \int_{0}^{t} \frac{(t-s)^{\alpha-1}}{\Gamma(\alpha)} v_{1}(s) d s-\frac{t}{3} \int_{0}^{1} \frac{(1-s)^{\alpha-1}}{\Gamma(\alpha)} v_{1}(s) d s \\
& -\frac{t}{3} \int_{0}^{1} \frac{(1-s)^{\alpha-2}}{\Gamma(\alpha-1)} v_{1}(s) d s+\left(t-t^{2}\right) \Delta \int_{0}^{1} \frac{(1-s)^{\alpha-3}}{\Gamma(\alpha-2)} v_{1}(s) d s \\
& +\left(t-t^{2}\right) \Delta \int_{0}^{1} \frac{(1-s)^{\alpha-p-1}}{\Gamma(\alpha-p)} v_{1}(s) d s, \quad t \in J .
\end{aligned}
$$

Hence,

$$
\begin{aligned}
& |z(t)| \leq\|p\|_{\infty}\left\{\frac{4}{3 \Gamma(\alpha+1)}+\frac{1}{3 \Gamma(\alpha)}+\frac{\Delta}{4 \Gamma(\alpha-1)}+\frac{\Delta}{4 \Gamma(\alpha-p+1)}\right\}, \\
& \left|z^{\prime}(t)\right| \leq\|p\|_{\infty}\left\{\frac{4}{3 \Gamma(\alpha)}+\frac{1}{3 \Gamma(\alpha+1)}+\frac{\Delta}{\Gamma(\alpha-1)}+\frac{\Delta}{\Gamma(\alpha-p+1)}\right\}, \\
& \left|z^{\prime \prime}(t)\right| \leq\|p\|_{\infty}\left\{\frac{1+2 \Delta}{\Gamma(\alpha-1)}+\frac{2 \Delta}{\Gamma(\alpha-p+1)}\right\}, \\
& \left|{ }^{c} D^{q_{i}} z(t)\right| \leq\|p\|_{\infty}\left\{\frac{1}{\Gamma\left(\alpha-q_{i}+1\right)}+\frac{2 \Delta}{\Gamma\left(3-q_{i}\right) \Gamma(\alpha-1)}+\frac{2 \Delta}{\Gamma\left(3-q_{i}\right) \Gamma(\alpha-p+1)}\right\}
\end{aligned}
$$

for $i=1, \ldots, k$. Hence, $\|z\| \leq\|p\|_{\infty}\left(\Lambda_{1}+\Lambda_{2}+\Lambda_{3}+\Lambda_{4}^{i}\right), i=1, \ldots, k$. 
Now, we show that $B$ maps $Y$ to equi-continuous subsets of $X$. Let $t_{1}, t_{2} \in J$ with $t_{1}<t_{2}$, $u \in Y$, and $z \in B(u)$. Choose $v_{1} \in S_{G, u}$ such that $z(t)$ is given by (2.9). Then we have

$$
\begin{aligned}
&\left|z\left(t_{2}\right)-z\left(t_{1}\right)\right| \leq\|p\|_{\infty}\left\{\frac{\left|t_{2}^{\alpha}-t_{1}^{\alpha}\right|}{\Gamma(\alpha+1)}+\frac{\left|t_{2}-t_{1}\right|}{3 \Gamma(\alpha+1)}+\frac{\left|t_{2}-t_{1}\right|}{3 \Gamma(\alpha)}+\frac{\Delta\left|\left[\left(t_{2}-t_{1}\right)-\left(t_{2}^{2}-t_{1}^{2}\right)\right]\right|}{\Gamma(\alpha-1)}\right. \\
&\left.+\frac{\Delta\left|\left[\left(t_{2}-t_{1}\right)-\left(t_{2}^{2}-t_{1}^{2}\right)\right]\right|}{\Gamma(\alpha-p+1)}\right\}, \\
&\left|z^{\prime}\left(t_{2}\right)-z^{\prime}\left(t_{1}\right)\right| \leq\|p\|_{\infty}\left\{\frac{\left|t_{2}^{\alpha-1}-t_{1}^{\alpha-1}\right|}{\Gamma(\alpha)}+\frac{2 \Delta\left|t_{2}-t_{1}\right|}{\Gamma(\alpha-1)}+\frac{2 \Delta\left|t_{2}-t_{1}\right|}{\Gamma(\alpha-p+1)}\right\}, \\
&\left|z^{\prime \prime}\left(t_{2}\right)-z^{\prime \prime}\left(t_{1}\right)\right| \leq\|p\|_{\infty} \frac{\left|t_{2}^{\alpha-2}-t_{1}^{\alpha-2}\right|}{\Gamma(\alpha-1)}
\end{aligned}
$$

and

$$
\begin{aligned}
& \left|{ }^{c} D^{q_{i}} z\left(t_{2}\right)-{ }^{c} D^{q_{i}} z\left(t_{1}\right)\right| \\
& \quad \leq\|p\|_{\infty}\left\{\frac{\left|t_{2}^{\alpha-q_{i}}-t_{1}^{\alpha-q_{i}}\right|}{\Gamma\left(\alpha-q_{i}+1\right)}+\frac{2 \Delta\left|t_{2}^{2-q_{i}}-t_{1}^{2-q_{i}}\right|}{\Gamma\left(3-q_{i}\right) \Gamma(\alpha-1)}+\frac{2 \Delta\left|t_{2}^{2-q_{i}}-t_{1}^{2-q_{i}}\right|}{\Gamma\left(3-q_{i}\right) \Gamma(\alpha-p+1)}\right\}
\end{aligned}
$$

for each $i=1, \ldots, k$. It is seen that the right-hand sides of the above inequalities tend to zero as $t_{2} \rightarrow t_{1}$. Hence, by using the Arzelá-Ascoli theorem, $B$ is compact.

Next, we prove that $B$ has a closed graph. Let $u_{n} \in Y$ and $z_{n} \in B\left(u_{n}\right)$ for all $n$ such that $u_{n} \rightarrow u_{0}$ and $z_{n} \rightarrow z_{0}$. We show that $z_{0} \in B\left(u_{0}\right)$. Associated with $z_{n} \in B\left(u_{n}\right)$ for each $n \in \mathbb{N}$, there exists $v_{n} \in S_{G, u_{n}}$ such that

$$
\begin{aligned}
z_{n}(t)= & \int_{0}^{t} \frac{(t-s)^{\alpha-1}}{\Gamma(\alpha)} v_{n}(s) d s-\frac{t}{3} \int_{0}^{1} \frac{(1-s)^{\alpha-1}}{\Gamma(\alpha)} v_{n}(s) d s \\
& -\frac{t}{3} \int_{0}^{1} \frac{(1-s)^{\alpha-2}}{\Gamma(\alpha-1)} v_{n}(s) d s+\left(t-t^{2}\right) \Delta \int_{0}^{1} \frac{(1-s)^{\alpha-3}}{\Gamma(\alpha-2)} v_{n}(s) d s \\
& +\left(t-t^{2}\right) \Delta \int_{0}^{1} \frac{(1-s)^{\alpha-p-1}}{\Gamma(\alpha-p)} v_{n}(s) d s
\end{aligned}
$$

for all $t \in J$. It suffices to show that there exists $v_{0} \in S_{G, u_{0}}$ such that, for each $t \in J$,

$$
\begin{aligned}
z_{0}(t)= & \int_{0}^{t} \frac{(t-s)^{\alpha-1}}{\Gamma(\alpha)} v_{0}(s) d s-\frac{t}{3} \int_{0}^{1} \frac{(1-s)^{\alpha-1}}{\Gamma(\alpha)} v_{0}(s) d s \\
& -\frac{t}{3} \int_{0}^{1} \frac{(1-s)^{\alpha-2}}{\Gamma(\alpha-1)} v_{0}(s) d s+\left(t-t^{2}\right) \Delta \int_{0}^{1} \frac{(1-s)^{\alpha-3}}{\Gamma(\alpha-2)} v_{0}(s) d s \\
& +\left(t-t^{2}\right) \Delta \int_{0}^{1} \frac{(1-s)^{\alpha-p-1}}{\Gamma(\alpha-p)} v_{0}(s) d s .
\end{aligned}
$$

Consider the continuous linear operator $\Theta: L^{1}(J, \mathbb{R}) \rightarrow X$ by

$$
\begin{aligned}
\Theta(v)(t)= & \int_{0}^{t} \frac{(t-s)^{\alpha-1}}{\Gamma(\alpha)} v(s) d s-\frac{t}{3} \int_{0}^{1} \frac{(1-s)^{\alpha-1}}{\Gamma(\alpha)} v(s) d s \\
& -\frac{t}{3} \int_{0}^{1} \frac{(1-s)^{\alpha-2}}{\Gamma(\alpha-1)} v(s) d s+\left(t-t^{2}\right) \Delta \int_{0}^{1} \frac{(1-s)^{\alpha-3}}{\Gamma(\alpha-2)} v(s) d s \\
& +\left(t-t^{2}\right) \Delta \int_{0}^{1} \frac{(1-s)^{\alpha-p-1}}{\Gamma(\alpha-p)} v(s) d s .
\end{aligned}
$$


Notice that

$$
\begin{aligned}
\left\|z_{n}(t)-z_{0}(t)\right\|= & \| \int_{0}^{t} \frac{(t-s)^{\alpha-1}}{\Gamma(\alpha)}\left(v_{n}(s)-v_{0}(s)\right) d s-\frac{t}{3} \int_{0}^{1} \frac{(1-s)^{\alpha-1}}{\Gamma(\alpha)}\left(v_{n}(s)-v_{0}(s)\right) d s \\
& -\frac{t}{3} \int_{0}^{1} \frac{(1-s)^{\alpha-2}}{\Gamma(\alpha-1)}\left(v_{n}(s)-v_{0}(s)\right) d s \\
& +\left(t-t^{2}\right) \Delta \int_{0}^{1} \frac{(1-s)^{\alpha-3}}{\Gamma(\alpha-2)}\left(v_{n}(s)-v_{0}(s)\right) d s \\
& +\left(t-t^{2}\right) \Delta \int_{0}^{1} \frac{(1-s)^{\alpha-p-1}}{\Gamma(\alpha-p)}\left(v_{n}(s)-v_{0}(s)\right) d s \| \rightarrow 0 \quad \text { as } n \rightarrow \infty .
\end{aligned}
$$

By using Lemma 1.2, $\Theta \circ S_{G}$ is a closed graph operator. Since $z_{n}(t) \in \Theta\left(S_{G, u_{n}}\right)$ for all $n$, and $u_{n} \rightarrow u_{0}$, there is $v_{0} \in S_{G, u_{0}}$ such that

$$
\begin{aligned}
z_{0}(t)= & \int_{0}^{t} \frac{(t-s)^{\alpha-1}}{\Gamma(\alpha)} v_{0}(s) d s-\frac{t}{3} \int_{0}^{1} \frac{(1-s)^{\alpha-1}}{\Gamma(\alpha)} v_{0}(s) d s \\
& -\frac{t}{3} \int_{0}^{1} \frac{(1-s)^{\alpha-2}}{\Gamma(\alpha-1)} v_{0}(s) d s+\left(t-t^{2}\right) \Delta \int_{0}^{1} \frac{(1-s)^{\alpha-3}}{\Gamma(\alpha-2)} v_{0}(s) d s \\
& +\left(t-t^{2}\right) \Delta \int_{0}^{1} \frac{(1-s)^{\alpha-p-1}}{\Gamma(\alpha-p)} v_{0}(s) d s .
\end{aligned}
$$

Hence, $z_{0} \in B\left(u_{0}\right)$. So, it follows that $B$ has a closed graph and this implies that the operator $B$ is upper semi-continuous.

Finally, we show that $A$ is a contraction multifunction. Let $u, w \in X$ and $z_{1} \in A(w)$ is given. Then we can select $v_{1} \in S_{F, w}$ such that

$$
\begin{aligned}
z_{1}(t)= & \int_{0}^{t} \frac{(t-s)^{\alpha-1}}{\Gamma(\alpha)} v_{1}(s) d s-\frac{t}{3} \int_{0}^{1} \frac{(1-s)^{\alpha-1}}{\Gamma(\alpha)} v_{1}(s) d s \\
& -\frac{t}{3} \int_{0}^{1} \frac{(1-s)^{\alpha-2}}{\Gamma(\alpha-1)} v_{1}(s) d s+\left(t-t^{2}\right) \Delta \int_{0}^{1} \frac{(1-s)^{\alpha-3}}{\Gamma(\alpha-2)} v_{1}(s) d s \\
& +\left(t-t^{2}\right) \Delta \int_{0}^{1} \frac{(1-s)^{\alpha-p-1}}{\Gamma(\alpha-p)} v_{1}(s) d s
\end{aligned}
$$

for all $t \in J$. Since

$$
\begin{aligned}
H_{d}\left(F\left(t, u(t), u^{\prime}(t), u^{\prime \prime}(t),{ }^{c} D^{q_{1}} u(t), \ldots,{ }^{c} D^{q_{k}} u(t)\right)\right. \\
\left.\quad-F\left(t, w(t), w^{\prime}(t), w^{\prime \prime}(t),{ }^{c} D^{q_{1}} w(t), \ldots,{ }^{c} D^{q_{k}} w(t)\right)\right) \\
\leq h(t)\left[|u(t)-w(t)|+\left|u^{\prime}(t)-w^{\prime}(t)\right|+\left|u^{\prime \prime}(t)-w^{\prime \prime}(t)\right|+\sum_{i=1}^{k}\left|{ }^{c} D^{q_{i}} u(t)-{ }^{c} D^{q_{i}} w(t)\right|\right]
\end{aligned}
$$

for almost all $t \in J$, there exists $y \in F\left(t, u(t), u^{\prime}(t), u^{\prime \prime}(t),{ }^{c} D^{q_{1}} u(t), \ldots,{ }^{c} D^{q_{k}} u(t)\right)$ such that

$$
\begin{aligned}
\left|v_{1}(t)-y\right| \leq & m(t)\left[|u(t)-w(t)|+\left|u^{\prime}(t)-w^{\prime}(t)\right|+\left|u^{\prime \prime}(t)-w^{\prime \prime}(t)\right|\right. \\
& \left.+\sum_{i=1}^{k}\left|{ }^{c} D^{q_{i}} u(t)-{ }^{c} D^{q_{i}} w(t)\right|\right]
\end{aligned}
$$


for almost all $t \in J$. Consider the multifunction $U: J \rightarrow \mathcal{P}(\mathbb{R})$ by

$$
U(t)=\left\{s \in \mathbb{R}:\left|v_{1}(t)-s\right| \leq m(t) g(t) \text { for almost all } t \in J\right\},
$$

where

$$
\begin{aligned}
g(t)= & {\left[|u(t)-w(t)|+\left|u^{\prime}(t)-w^{\prime}(t)\right|+\left|u^{\prime \prime}(t)-w^{\prime \prime}(t)\right|\right.} \\
& \left.+\sum_{i=1}^{k}\left|{ }^{c} D^{q_{i}} u(t)-{ }^{c} D^{q_{i}} w(t)\right|\right] .
\end{aligned}
$$

Since $v_{1}$ and $\varphi=m g$ are measurable, $U(\cdot) \cap F\left(\cdot, u(\cdot), u^{\prime}(\cdot), u^{\prime \prime}(\cdot),{ }^{c} D^{q_{1}} u(\cdot), \ldots,{ }^{c} D^{q_{k}} u(\cdot)\right)$ is a measurable multifunction. Thus, we can choose

$$
v_{2}(t) \in F\left(t, u(t), u^{\prime}(t), u^{\prime \prime}(t),{ }^{c} D^{q_{1}} u(t), \ldots,{ }^{c} D^{q_{k}} u(t)\right)
$$

such that

$$
\begin{aligned}
\left|v_{1}(t)-v_{2}(t)\right| \leq & m(t)\left[|u(t)-w(t)|+\left|u^{\prime}(t)-w^{\prime}(t)\right|+\left|u^{\prime \prime}(t)-w^{\prime \prime}(t)\right|\right. \\
& \left.+\sum_{i=1}^{k}\left|{ }^{c} D^{q_{i}} u(t)-{ }^{c} D^{q_{i}} w(t)\right|\right]
\end{aligned}
$$

and

$$
\begin{aligned}
z_{2}(t)= & \int_{0}^{t} \frac{(t-s)^{\alpha-1}}{\Gamma(\alpha)} v_{2}(s) d s-\frac{t}{3} \int_{0}^{1} \frac{(1-s)^{\alpha-1}}{\Gamma(\alpha)} v_{2}(s) d s \\
& -\frac{t}{3} \int_{0}^{1} \frac{(1-s)^{\alpha-2}}{\Gamma(\alpha-1)} v_{2}(s) d s+\left(t-t^{2}\right) \Delta \int_{0}^{1} \frac{(1-s)^{\alpha-3}}{\Gamma(\alpha-2)} v_{2}(s) d s \\
& +\left(t-t^{2}\right) \Delta \int_{0}^{1} \frac{(1-s)^{\alpha-p-1}}{\Gamma(\alpha-p)} v_{2}(s) d s
\end{aligned}
$$

for all $t \in J$. Now, we have

$$
\begin{aligned}
\left|z_{1}(t)-z_{2}(t)\right| \leq & \frac{1}{\Gamma(\alpha)} \int_{0}^{t}(t-s)^{\alpha-1}\left|v_{1}(s)-v_{2}(s)\right| d s \\
& +\frac{|t|}{3 \Gamma(\alpha)} \int_{0}^{1}(1-s)^{\alpha-1}\left|v_{1}(s)-v_{2}(s)\right| d s \\
& +\frac{|t|}{3 \Gamma(\alpha-1)} \int_{0}^{1}(1-s)^{\alpha-2}\left|v_{1}(s)-v_{2}(s)\right| d s \\
& +\frac{\Delta\left|t-t^{2}\right|}{\Gamma(\alpha-2)} \int_{0}^{1}(1-s)^{\alpha-3}\left|v_{1}(s)-v_{2}(s)\right| d s \\
& +\frac{\Delta\left|t-t^{2}\right|}{\Gamma(\alpha-p)} \int_{0}^{1}(1-s)^{\alpha-p-1}\left|v_{1}(s)-v_{2}(s)\right| d s \\
\leq & \|h\|_{\infty}\left\{\frac{4}{3 \Gamma(\alpha+1)}+\frac{1}{3 \Gamma(\alpha)}+\frac{\Delta}{4 \Gamma(\alpha-1)}+\frac{\Delta}{4 \Gamma(\alpha-p+1)}\right\}\|u-w\| .
\end{aligned}
$$


Similarly,

$$
\begin{aligned}
& \left|z_{1}^{\prime}(t)-z_{2}^{\prime}(t)\right| \leq\|h\|_{\infty}\left\{\frac{4}{3 \Gamma(\alpha)}+\frac{1}{3 \Gamma(\alpha+1)}+\frac{\Delta}{\Gamma(\alpha-1)}+\frac{\Delta}{\Gamma(\alpha-p+1)}\right\}\|u-w\|, \\
& \left|z_{1}^{\prime \prime}(t)-z_{2}^{\prime \prime}(t)\right| \leq\|h\|_{\infty}\left\{\frac{1+2 \Delta}{\Gamma(\alpha-1)}+\frac{2 \Delta}{\Gamma(\alpha-p+1)}\right\}\|u-w\|, \\
& \left|{ }^{c} D^{q_{i}} z_{1}(t)-{ }^{c} D^{q_{i}} z_{2}(t)\right| \\
& \quad \leq\|h\|_{\infty}\left\{\frac{1}{\Gamma\left(\alpha-q_{i}+1\right)}+\frac{2 \Delta}{\Gamma\left(3-q_{i}\right) \Gamma(\alpha-1)}+\frac{2 \Delta}{\Gamma\left(3-q_{i}\right) \Gamma(\alpha-p+1)}\right\}\|u-w\| .
\end{aligned}
$$

Hence,

$$
\begin{aligned}
& \sup _{t \in J}\left|z_{1}(t)-z_{2}(t)\right| \leq\|h\|_{\infty} \Lambda_{1}\|u-w\|, \\
& \sup _{t \in J}\left|z_{1}^{\prime}(t)-z_{2}^{\prime}(t)\right| \leq\|h\|_{\infty} \Lambda_{2}\|u-w\|, \\
& \sup _{t \in J}\left|z_{1}^{\prime \prime}(t)-z_{2}^{\prime \prime}(t)\right| \leq\|h\|_{\infty} \Lambda_{3}\|u-w\|, \\
& \sup _{t \in J}\left|{ }^{c} D^{q_{i}} z_{1}(t)-{ }^{c} D^{q_{i}} z_{2}(t)\right| \leq\|h\|_{\infty} \Lambda_{4}^{i}\|u-w\|
\end{aligned}
$$

for each $1 \leq i \leq k$. So

$$
\left\|z_{1}-z_{2}\right\| \leq\|h\|_{\infty}\left(\Lambda_{1}+\Lambda_{2}+\Lambda_{3}+\Lambda_{4}^{i}\right)\|u-w\|, \quad i=1,2, \ldots, k .
$$

This implies that $H_{d}(A(u), A(w)) \leq L\|u-w\|$. Thus $A$ and $B$ satisfy all the conditions of Theorem 1.3 and so the inclusion $u \in A(u)+B(u)$ has a solution in $Y$. Therefore the inclusion problem (1.1)-(1.2) has a solution in $Y$ and the proof is completed.

Finally, we give an example to illustrate the validity of our main result.

Example 2.5 Consider the following fractional differential inclusion:

$$
\begin{aligned}
{ }^{c} D^{\frac{5}{2}} u(t) \in & {\left[0, \frac{t|u(t)|^{3}}{100\left(1+|u(t)|^{3}\right)}+\frac{t\left|2 \sin \left(u^{\prime}(t)\right)\right|}{200\left(\left|\sin \left(u^{\prime}(t)\right)\right|+1\right)}+\frac{0.01 t\left|u^{\prime \prime}(t)\right|}{\left|u^{\prime \prime}(t)\right|+1}\right.} \\
& \left.+\frac{t\left|\cos \left({ }^{c} D^{\frac{3}{2}} u(t)\right)\right|}{100\left(1+\left|\cos \left({ }^{c} D^{\frac{3}{2}} u(t)\right)\right|\right)}+\frac{\left.\left.t^{2}\left|\sin \frac{\pi}{2} t\right|\right|^{c} D^{\frac{3}{2}} u(t)\right|^{2}}{100 t\left(\left|D^{c} D^{\frac{3}{2}} u(t)\right|^{2}+1\right)}\right] \\
& +\left[0, \frac{e^{-t}|u(t)|}{\left(1+e^{t}\right)(1+|u(t)|)}+\frac{|\cos \pi t|\left|u^{\prime}(t)\right| e^{-t}}{\left(1+e^{t}\right)\left(1+\left|u^{\prime}(t)\right|\right)}+\frac{e^{-t}\left|u^{\prime \prime}(t)\right|^{2}}{\left(1+\left|u^{\prime \prime}(t)\right|^{2}\right)\left(1+e^{t}\right)}\right. \\
& \left.+\frac{e^{-2 t}\left|\sin \left({ }^{c} D^{\frac{3}{2}} u(t)\right)\right|}{\left(1+\left|\sin \left({ }^{c} D^{\frac{3}{2}} u(t)\right)\right|\right) e^{t}\left(1+e^{t}\right)}+\frac{e^{-3 t}\left|{ }^{c} D^{\frac{3}{2}} u(t)\right|^{3}}{\left(e^{2 t}+e^{3 t}\right)\left(1+\left|{ }^{c} D^{\frac{3}{2}} u(t)\right|^{3}\right)}\right],
\end{aligned}
$$

with the following boundary conditions:

$$
u(0)=0, \quad u^{\prime}(0)=-u(1)-u^{\prime}(1), \quad u^{\prime \prime}(0)=-u^{\prime \prime}(1)-{ }^{c} D^{\frac{3}{2}} u(1),
$$

where $t \in[0,1]$. In the above inclusion problem, we have $\alpha=5 / 2, p=3 / 2, k=2$, and $q_{1}=$ $q_{2}=3 / 2$. Also, we have $\Delta=0.1597$. 
Now, we define $F:[0,1] \times \mathbb{R} \times \mathbb{R} \times \mathbb{R} \times \mathbb{R} \times \mathbb{R} \rightarrow \mathcal{P}(\mathbb{R})$ by

$$
\begin{aligned}
F(t, x, y, z, v, w)= & {\left[0, \frac{t|x|^{3}}{100\left(1+|x|^{3}\right)}+\frac{t|2 \sin y|}{200(|\sin y|+1)}+\frac{0.01 t|z|}{|z|+1}\right.} \\
& \left.+\frac{t|\cos v|}{100(1+|\cos v|)}+\frac{t^{2}\left|\sin \frac{\pi}{2} t\right| w^{2}}{100 t\left(w^{2}+1\right)}\right],
\end{aligned}
$$

and $G:[0,1] \times \mathbb{R} \times \mathbb{R} \times \mathbb{R} \times \mathbb{R} \times \mathbb{R} \rightarrow \mathcal{P}(\mathbb{R})$ by

$$
\begin{aligned}
G(t, x, y, z, v, w)= & {\left[0, \frac{e^{-t}|x|}{\left(1+e^{t}\right)(1+|x|)}+\frac{|\cos \pi t||y| e^{-t}}{\left(1+e^{t}\right)(1+|y|)}+\frac{e^{-t} z^{2}}{\left(1+z^{2}\right)\left(1+e^{t}\right)}\right.} \\
& \left.+\frac{e^{-2 t}|\sin v|}{(1+|\sin v|) e^{t}\left(1+e^{t}\right)}+\frac{e^{-3 t}|w|^{3}}{\left(e^{2 t}+e^{3 t}\right)\left(1+|w|^{3}\right)}\right]
\end{aligned}
$$

Then there exist continuous functions $m, p:[0,1] \rightarrow(0, \infty)$ given by

$$
m(t)=5+\frac{t}{100}, \quad p(t)=\frac{e^{-t}}{1+e^{t}}
$$

On the other hand, we can easily check that, for every $x_{i}, y_{i}, z_{i}, v_{i}, w_{i} \in \mathbb{R}(i=1,2)$,

$$
\begin{aligned}
& H_{d}\left(F\left(t, x_{1}, y_{1}, z_{1}, v_{1}, w_{1}\right)-F\left(t, x_{2}, y_{2}, z_{2}, v_{2}, w_{2}\right)\right) \\
& \quad \leq h(t)\left(\left|x_{1}-x_{2}\right|+\left|y_{1}-y_{2}\right|+\left|z_{1}-z_{2}\right|+\left|v_{1}-v_{2}\right|+\left|w_{1}-w_{2}\right|\right),
\end{aligned}
$$

where $h:[0,1] \rightarrow(0, \infty)$ is defined by $h(t)=\frac{t}{100}$. It can easily be found that $\Lambda_{1}=0.7369$, $\Lambda_{2}=1.4434, \Lambda_{3}=1.8102, \Lambda_{4}^{1}=1.7687$, and $\Lambda_{4}^{2}=1.7687$. Since $\|h\|_{\infty}=\frac{1}{100}$, we have $L:=\|h\|_{\infty}\left(\Lambda_{1}+\Lambda_{2}+\Lambda_{3}+\Lambda_{4}^{1}+\Lambda_{4}^{2}\right)=0.01 \times 7.5279=0.075279<1$. Consequently all assumptions and conditions of Theorem 2.4 are satisfied. Hence, Theorem 2.4 implies that the fractional differential inclusion problem (2.10)-(2.11) has a solution.

\section{Competing interests}

The authors declare that they have no competing interests.

\section{Authors' contributions}

All authors contributed equally in this article. They read and approved the final manuscript.

\section{Author details}

${ }^{1}$ Department of Mathematics, University of loannina, loannina, 451 10, Greece. ${ }^{2}$ Nonlinear Analysis and Applied Mathematics (NAAM) Research Group, Department of Mathematics, Faculty of Science, King Abdulaziz University, P.O. Box 80203, Jeddah, 21589, Saudi Arabia. ${ }^{3}$ Young Researchers and Elite Club, Tabriz Branch, Islamic Azad University, Tabriz, Iran. ${ }^{4}$ Nonlinear Dynamic Analysis Research Center, Department of Mathematics, Faculty of Applied Science, King Mongkut's University of Technology North Bangkok, Bangkok, 10800, Thailand.

\section{Acknowledgements}

We would like to thank the reviewers for their valuable comments and suggestions on the manuscript.

Received: 20 January 2015 Accepted: 21 April 2015 Published online: 06 May 2015

\section{References}

1. Agarwal, RP, Ahmad, B: Existence theory for anti-periodic boundary value problems of fractional differential equations and inclusions. J. Appl. Math. Comput. 62, 1200-1214 (2011)

2. Agarwal, RP, Belmekki, M, Benchohra, M: A survey on semilinear differential equations and inclusions involving Riemann-Liouville fractional derivative. Adv. Differ. Equ. 2009, Article ID 981728 (2009)

3. Agarwal, RP, Benchohra, M, Hamani, S: A survey on existence results for boundary value problems of nonlinear fractional differential equations and inclusions. Acta Appl. Math. 109, 973-1033 (2010) 
4. Agarwal, RP, Ntouyas, SK, Ahmad, B, Alhothuali, M: Existence of solutions for integro-differential equations of fractional order with nonlocal three-point fractional boundary conditions. Adv. Differ. Equ. 2013, 128 (2013)

5. Ahmad, B, Nieto, JJ: Existence of solutions for anti-periodic boundary value problems involving fractional differential equations via Leray-Schauder degree theory. Topol. Methods Nonlinear Anal. 35, 295-304 (2010)

6. Ahmad, B, Ntouyas, SK: Boundary value problem for fractional differential inclusions with four-point integral boundary conditions. Surv. Math. Appl. 6, 175-193 (2011)

7. Ahmad, B, Ntouyas, SK, Alsedi, A: On fractional differential inclusions with anti-periodic type integral boundary conditions. Bound. Value Probl. 2013, 82 (2013)

8. Alsaedi, A, Ntouyas, SK, Ahmad, B: Existence of solutions for fractional differential inclusions with separated boundary conditions in Banach space. Abstr. Appl. Anal. 2013, Article ID 869837 (2013)

9. Bai, Z, Sun, W: Existence and multiplicity of positive solutions for singular fractional boundary value problems. Comput. Math. Appl. 63, 1369-1381 (2012)

10. Baleanu, D, Agarwal, RP, Mohammadi, H, Rezapour, S: Some existence results for a nonlinear fractional differential equation on partially ordered Banach spaces. Bound. Value Probl. 2013, 112 (2013)

11. Baleanu, D, Diethelm, K, Scalas, E, Trujillo, JJ: Fractional Calculus Models and Numerical Methods. World Scientific, Singapore (2012)

12. Baleanu, D, Mohammadi, $H$, Rezapour, S: The existence of solutions for a nonlinear mixed problem of singular fractional differential equations. Adv. Differ. Equ. 2013, 359 (2013)

13. Baleanu, D, Mohammadi, H, Rezapour, S: Positive solutions of a boundary value problem for nonlinear fractional differential equations. Abstr. Appl. Anal. 2012, Article ID 837437 (2012)

14. Baleanu, D, Mohammadi, H, Rezapour, S: Some existence results on nonlinear fractional differential equations. Philos. Trans. R. Soc. Lond. A 371, 20120144 (2013)

15. Baleanu, D, Mohammadi, H, Rezapour, S: On a nonlinear fractional differential equation on partially ordered metric spaces. Adv. Differ. Equ. 2013, 83 (2013)

16. Baleanu, D, Nazemi, Z, Rezapour, S: The existence of positive solutions for a new coupled system of multi-term singular fractional integro-differential boundary value problems. Abstr. Appl. Anal. 2013, Article ID 368659 (2013)

17. Baleanu, D, Nazemi, Z, Rezapour, S: Existence and uniqueness of solutions for multi-term nonlinear fractional integro-differential equations. Adv. Differ. Equ. 2013, 368 (2013)

18. Baleanu, D, Nazemi, Z, Rezapour, S: Attractivity for a $k$-dimensional system of fractional functional differential equations and global attractivity for a $k$-dimensional system of nonlinear fractional differential equations. J. Inequal. Appl. 2014, 31 (2014)

19. Bragdi, M, Debbouche, A, Baleanu, D: Existence of solutions for fractional differential inclusions with separated boundary conditions in Banach space. Adv. Math. Phys. 2013, Article ID 426061 (2013)

20. Campos, LM: On the solution of some simple fractional differential equations. Int. J. Math. Sci. 13(3), 481-496 (1990)

21. Luchko,, , Srivastava, $\mathrm{H}$ : The exact solution of certain differential equations of fractional order by using operational calculus. Comput. Math. Appl. 29, 73-85 (1995)

22. Ouahab, A: Some results for fractional boundary value problem of differential inclusions. Nonlinear Anal. 69, 3877-3896 (2008)

23. Podlubny, I: Fractional Differential Equations. Academic Press, San Diego (1999)

24. Phung, $P D$, Truong, LX: On a fractional differential inclusion with integral boundary conditions in Banach space. Fract. Calc. Appl. Anal. 16, 538-558 (2013)

25. Samko, G, Kilbas, A, Marichev, O: Fractional Integrals and Derivatives: Theory and Applications. Gordon \& Breach, New York (1993)

26. Diethelm, K: Analysis of Fractional Differential Equations. Springer, Berlin (2010)

27. Oldham, KB, Spanier, J: The Fractional Calculus. Academic Press, San Diego (1974)

28. Miller, S, Ross, B: An Introduction to the Fractional Calculus and Fractional Differential Equations. Wiley, New York (1993)

29. Deimling, K: Multi-Valued Differential Equations. de Gruyter, Berlin (1992)

30. Hu, S, Papageorgiou, N: Handbook of Multivalued Analysis. Volume I: Theory. Kluwer Academic, Dordrecht (1997)

31. Aubin, J, Ceuina, A: Differential Inclusions: Set-Valued Maps and Viability Theory. Springer, Berlin (1984)

32. Lasota, A, Opial, Z: An application of the Kakutani-Ky Fan theorem in the theory of ordinary differential equations. Bull. Acad. Pol. Sci., Sér. Sci. Math. Astron. Phys. 13, 781-786 (1965)

33. Petrusel, A: Fixed points and selections for multi-valued operators. Semin. Fixed Point Theory Cluj-Napoca 2, 3-22 (2001)

34. Su, X: Boundary value problem for a coupled system of nonlinear fractional differential equations. Appl. Math. Lett. $22,64-69$ (2009)

35. Ibrahim, AG: Fractional differential inclusions with anti-periodic boundary conditions in Banach spaces. Electron. J. Qual. Theory Differ. Equ. 2014, 65 (2014)

36. Lan, Q, Lin, W: Positive solutions of systems of Caputo fractional differential equations. Commun. Appl. Anal. 17, 61-86 (2013) 